\title{
BMJ Open Defining the concepts of a smart nursing home and its potential technology utilities that integrate medical services and are acceptable to stakeholders: a scoping review protocol
}

\author{
Yuanyuan Zhao (D) ,, Fakhrul Zaman Rokhani (D) ,", Sazlina Shariff Ghazali (D) ,1,4 \\ Boon How Chew (i) 1,5
}

To cite: Zhao Y, Rokhani FZ, Shariff Ghazali S, et al. Defining the concepts of a smart nursing home and its potential technology utilities that integrate medical services and are acceptable to stakeholders: a scoping review protocol. BMJ Open 2021;11:e041452. doi:10.1136/ bmjopen-2020-041452

- Prepublication history and supplemental material for this paper is available online. To view these files, please visit the journal online (http://dx.doi. org/10.1136/bmjopen-2020 041452).

Received 10 June 2020 Revised 07 January 2021 Accepted 19 January 2021

Check for updates

(C) Author(s) (or their employer(s)) 2021. Re-use permitted under CC BY-NC. No commercial re-use. See rights and permissions. Published by BMJ.

For numbered affiliations see end of article.

Correspondence to Dr Boon How Chew; chewboonhow@upm.edu.my

\section{ABSTRACT}

Introduction Smart technologies, digital health and eHealth have been shown to enhance institutional elderly care. Because of the rapidly ageing societies, information technologies in geriatric healthcare are urgently needed. A lot of innovation in smart healthcare has occurred in the past decade, and its use in nursing care assessment, daily living activities and service management is yet to be defined. More fundamentally, the concepts, definitions and scopes of a smart nursing home are still vague. Thus, this scoping review aims to examine the extent, range (variety) and nature (characteristics) of evidence on the existing smart concepts and feasible healthcare technologies, types of medical services in nursing home settings and acceptability of a smart nursing home by the elderly people $\geq 60$ years old, their caregivers, nursing home operators and government agencies.

Methods and analysis This scoping review will be guided by the smart technology adoption behaviours of elder consumers theoretical model (Elderadopt) by Golant and reported according to the Preferred Reporting Items for Systematic Reviews and Meta-Analyses for Scoping Reviews. First, we will conduct an internet search for nursing homes and websites and databases related to the stakeholders to retrieve the definitions, concepts and criteria of a smart nursing home (phase 1). Second, we will conduct an additional systematic electronic database search for published articles on any measures of technological feasibility and integration of medical services in nursing home settings and their acceptability by nursing home residents and caregivers (phase 2). The electronic database search will be carried out from 1999 to 30 September 2020 and limited to works published in English and Chinese languages. For phase 2, the selection of literature is further limited to residents of nursing homes aged $\geq 60$ years old with or without medical needs but are not terminally ill or bed-bound. Qualitative data analysis will follow the Framework Methods and thematic analysis using combined inductive and deductive approaches, conducted by at least two reviewers.

Ethics and dissemination This protocol is registered on osf.io (URL: https://osf.io/qtwz2/). Ethical approval is not necessary as the scoping review is not a primary study,

\section{Strengths and limitations of this study}

To the best of our knowledge, this is the first scoping review to identify the extent of the evidence and gaps in the literature on the concept of a smart nursing home, information technologies and integration of medical services in nursing homes and their acceptability.

- The review would contribute to a better smart nursing home business model for the rapidly ageing societies in developed and low-income and middleincome countries, including China.

- Comprehensive search through the Internet, government documents and electronic databases will be conducted, and textual data will be analysed qualitatively.

- The search and review will be limited to the literature published in English and Chinese.

- The results may be relevant to a broad base of readers interested in this research and to most industrialised countries with nursing homes, and the elderly people living in them.

and the information is collected from selected articles that are publicly available sources. All findings will be disseminated at conferences and published in peerreviewed journals.

\section{INTRODUCTION}

The term 'smart' in healthcare-related industries is widely associated with smart health, smart healthcare, smart medicine, smart hospitals and smart home care. The term 'smart' originated from International Business Machines Corporation concept of 'Smarter Planet'. 'WHO (2019) associates smart health with telemedicine, mobile applications for health services and digital health. The term 'digital health' is a broad umbrella term encompassing eHealth which also includes mHealth, as well as in emerging 
areas such as in advanced computing sciences of big data and artificial intelligence (AI). ${ }^{2}$

Earlier, smart healthcare was labelled as 'ubiquitous healthcare' (u-healthcare), to describe an emerging area of technology that uses a large number of environmental and patient sensors and actuators in monitoring physical and mental conditions of patients. ${ }^{3}$ Ubiquitous healthcare envisions sensor networks and the Internet of Things (IoT) to improve health-related services. ${ }^{45}$ With the development of information technology (IT), Tian et at proposed a comprehensive definition and summarised the applications of smart healthcare that use a new generation of information technologies such as the loT, big data, cloud computing and AI to transform the traditional medical systems and make healthcare more efficient, convenient and personalised. Smart healthcare applications are commonly used in disease prevention and monitoring, diagnosis and treatment of health conditions, hospital management, health decision making and medical research.

Smart ageing is an emerging research topic that has a profound impact on the society and well-being of ageing population. ${ }^{7}$ In 2019, the rising number of elderly people aged $\geq 65$ years old has reached 703 million worldwide. ${ }^{8}$ Elderly people are often assumed to be frail or dependent and a burden to the society. ${ }^{9}$ They may require a wide range of assistance in tasks such as physical mobility, monitoring, providing reminders or social interaction. ${ }^{10}$ One such challenge is providing the elderly with realtime, long-term and nonintrusive assisted living and remote healthcare services. ${ }^{11}$ Klimova ${ }^{12}$ mentioned that smart healthcare with modern information and communication technologies, particularly the 'smart' ones, has the capacity to meet the needs of the elderly people so that they are able to lead an active, fulfilling and quality life. Sundaravadivel $e t a l^{13}$ indicated that smart healthcare using IoT could be implemented at all levels in institutional care and home-based care, especially in elderly care. IoT helps use available resources to their maximum potential without any geographical barriers; for example, in aiding remote monitoring of patients and disease management, and improving access to healthcare resources for the elderly people while reducing strain on healthcare systems. ${ }^{14}$ The 'Emerging Technologies to Support an Ageing Population' report issued by the National Science \& Technology Council, an American committee on technology, in 2019 encourages innovation in real-time monitoring of chronic conditions, mobile phone-based medical interventions and healthcare assistance, electronic health record, clinical decision supports and remote health system integration for elderly care. ${ }^{15}$ Several studies have shown that smart technologies are feasible in home-based care to enable ageing in place, and smart technologies make it possible to monitor and provide care for home residents in distance. This will enhance the quality of their life and promote independent living. ${ }^{16}$ Moreover, advanced technology such as the intelligent sensor system can be an effective strategy to detect and intervene in early signs of functional decline and early illness so that the elderly people can get help or treatment early. ${ }^{17}$ The wireless sensor network system also makes it feasible to supervise a high number of elderly people in home-based care and institutional care. ${ }^{18}$

Several countries, including China, are facing complex challenges of rapid ageing. ${ }^{19}$ According to China's National Laws, published in 2012, elderly people are defined as those aged $\geq 60$ years old. ${ }^{20}$ The United Nations defines a population as an ageing society when $>7 \%$ of the total population is $\geq 65$ years old, an aged society when $>14 \%$ of the total population is $\geq 65$ years old and a super-aged society or postaged society when $>20 \%$ of the total population is $\geq 65$ years old. The statistic from the National Bureau of Statistics of China (2018) revealed that the number of elderly people aged $\geq 65$ years old had reached 167 million (11.9\%) in 2018, and predicted that China would become a super-aged society with more than $21 \%$ elderly population by $2035 .^{21}$ By contrast, France took 100 years to progress from an ageing society $(7 \%)$ to the aged society $(14 \%) .{ }^{22}$ In 2019 , up to $75.8 \%$ of the Chinese elderly people aged $\geq 60$ years old had at least one chronic disease. ${ }^{23}$ The population aged $\geq 80$ years old has doubled the number of those aged $\geq 65$ years old, and their medical demand is five times higher than those between 65 and 79 years. ${ }^{24}$ Additionally, influenced by the Confucian philosophy of filial piety, a virtue 'Xiao' and respect for one's parents and the elders,${ }^{25}$ most Chinese and Asian elderly people prefer 'ageing in place' and growing old in their homes or spending the rest of their life with their children. ${ }^{26}$ However, it has become hardly feasible for a single child or grandchild to care for two parents or four grandparents in China's one-child policy that produces a family structure of ' $4-2-1$ '. 'Ageing in place' is desirable; however, the elderly people may expect a transition from home-based care to institutional care facilities with an intensive supervision due to changes in health status, mental health issues and impediment for family members who are unable to devote themselves to the necessary or additional care.$^{27}$ Fortunately, more elderly people in China are accepting nursing homes as an alternative to ageing in their homes. ${ }^{28}$ A nursing home provides a 24-hour functional care and some degree of medical supports from healthcare professionals for the elderly people who require assistance with activities of daily living and who often have complex health needs. ${ }^{29}$ Admission to a nursing home is usually a major life event for most elderly people. ${ }^{30}$

The demand for nursing homes is huge and is expected to increase, which is not met by the existing nursing homes and business models in the future. In 2015, the Chinese government reported that there were approximately 5.5 million nursing beds, an average of 26 beds per 1000 elderly people. ${ }^{31}$ This is clearly inadequate knowing that the estimated number of elderly people in the country is 30 times more. ${ }^{32}$ In addition, the quality of care and medical services available in most nursing homes are largely suboptimal. Many elderly residents in 
nursing homes have to visit hospitals for their primary medical care. ${ }^{33}$ The ageing population may affect the country's economy, society, policy and culture if they are not properly managed. ${ }^{34}$ To cope with the challenges related to bed capacity at nursing homes, the Chinese government has proposed a national plan based on home care, relying on the society and supporting the institutions for the elderly care, ${ }^{35}$ and initiated 'The Healthy China 2030' to address integration of medical resources to better serve the senior population. ${ }^{36}$ Smart technologies empower the elderly people to live independently and reduce the burden of the family caregivers in monitoring, controlling and supporting their loved ones at home. ${ }^{37}$ Evidence has shown that nursing homes that integrate smart technologies also benefit health professionals by reducing unnecessary workload and saving time in providing better medical services for nursing home residents, ${ }^{38} 39$ such as by using telepresence assistance and wearable devices, and integrating hospitals to overcome the needs in institutionalised care of the elderly people with chronic conditions. ${ }^{40}$

To facilitate a clear concept for implementing smart technologies and the advantages in nursing home settings, a scoping review is needed to bridge the gap between the definition of a smart nursing home, the availability and challenges of using information technologies in geriatric healthcare to meet the demands and aspirations of the rapidly growing elderly people, and to identify a suitable future nursing home model for the developed and lowincome and middle-income countries, including China. It is believed that a lot of innovation in smart healthcare with the proposed software and hardware entities can be used to promote nursing care in health assessment, daily living activities and care management that improve the quality of care and safety at nursing homes. ${ }^{42}$ This has been shown by the IoT-based smart devices and systems that interconnect medical resources to provide reliable and effective medical services to elderly people, especially those with chronic illnesses. ${ }^{43}$ In addition to enhancing the quality of existing services, smart healthcare could reduce healthcare costs through more efficient use of resources. $^{44-46}$ Therefore, standardising the definition and scope or level of smart nursing homes would help future initiatives and introduction of smart healthcare technologies that would allow the evaluation and monitoring of the operation at smart nursing homes.

\section{Theoretical framework}

We will adopt the smart technology adoption behaviours of elder consumers theoretical model by Golant and SMJJoAS ${ }^{47}$ to guide this scoping review (figure 1). ${ }^{47}$ This model offers adequate explanation on the behaviours of the elderly people with regard to the use of new technologies, as well as on the caregivers' perspectives during the appraisal of the technologies to be used in the care of the elderly people. The elderly people who have greater resilience and have stronger perceptions of self-efficacy and greater openness to new information will be more likely to evaluate the external information of the technologies (persuasion of outside messaging sources) before coming to the final adoption. This behavioural process is influenced by their past experiences (internal information). For the persuasiveness of the external information to be possible, a clear concept on a smart nursing home is necessary. According to the theoretical model, the caregivers of elderly people would experience similar coping processes from the appraisal of the needs for a smart technology to feeling about the persuasiveness of the external information such as from friends, colleagues or other healthcare professionals pertaining to the technology, perceived efficaciousness and usability until the stage of coming to a decision of whether to adopt the 'new' smart technology or maintain the 'old' ways of life, after putting all ideas in order. Therefore, the coping processes may occur in the non-elderly stakeholder's own perspectives or feeling about unmet needs of the elderly people in coping with smart technologies. Technology appraisals are feasible when the range of technologies have been proven to be applicable and safe in nursing homes. This includes smart technologies that integrate medical services and are deemed secured from personal privacy breaches. Acceptability of these features by all smart nursing home stakeholders will also be affected by other factors such as cost, quality of care, general ambience and vicinity of the nursing home to family members. ${ }^{48}$ Hence, this model is appropriate in guiding the formulation of the review questions, objectives, data extraction and analysis and in offering the explanatory framework to the discussion of the review findings.

\section{Review questions and objectives}

This scoping review will be conducted to systematically map the existing smart concepts and smart healthcare technologies within nursing home settings. We will

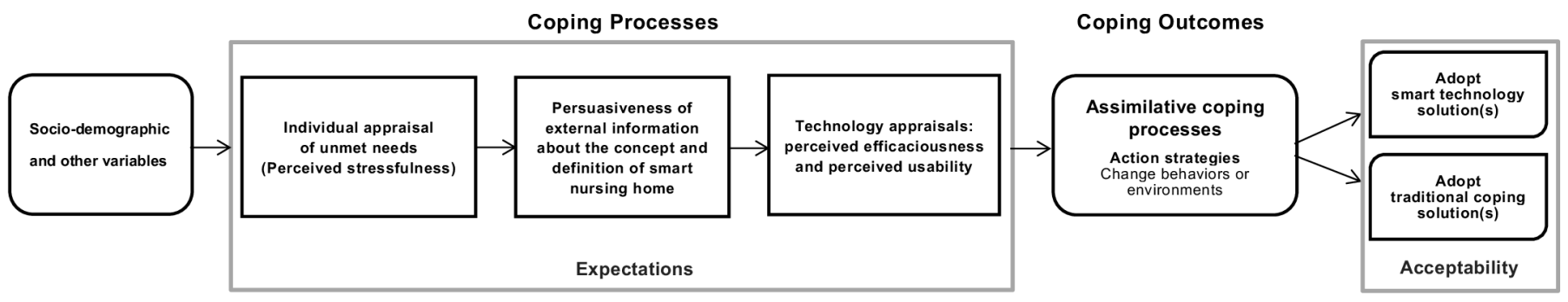

Figure 1 The theoretical framework for the scoping review. 
summarise findings from a body of knowledge that is heterogeneous in disciplines to identify the gaps in literature to aid the planning and commissioning of future research. This scoping review will be guided by Preferred Reporting Items for Systematic Reviews and Meta-Analyses Extension for Scoping Reviews. ${ }^{49}$

The following review questions are formulated: What is the definition of a smart nursing home? What is the availability of information technologies? What are the challenges of using information technologies? How will information technologies meet the demands and aspirations of the elderly end users and respective stakeholders? How can smart technology/information technology be integrated into a care delivery model in aged care? This proposed scoping review aims to examine the extent (size), range (variety) and nature (characteristics) of the evidence on technological feasibility, integration of medical services and acceptability of a smart nursing home by all stakeholders (the elderly people, caregivers, nursing homes operators and government agencies).

In this scoping review, a nursing home is defined as 'a facility with a home-like environment that provides a 24-hour nursing care for elderly people who require assistance with daily living activities and who often have complex health needs'. Nursing homes also provide some degree of supports from healthcare professionals providing primary medical services within the institutions. Residency in a nursing home may be relatively brief for respite purposes, for example, those requiring long-term, palliative/hospice and end-of-life care. $^{29}$ The framework of a smart nursing home is expected to include system design, IT solutions and architectures for running a smart nursing home such as the architecture of big data input and analysis. Elderly people are defined as those aged 60 years old and above. ${ }^{20}$ The stakeholders of a nursing home include the elderly people and their family members, healthcare professionals, nursing home administrators and department supervisors. ${ }^{50-63}$ In mainland China, the department supervisor for nursing homes is the Ministry of Civil Affairs. Aspects described as integral parts of a smart nursing home include adherence to guidelines, standards and other elements relating to the evaluation of a smart nursing home. The integration of medical services means integrating complex health services as a health network that could supplement the lack of coordination so that clinical efficiency can be achieved, and generating equal opportunities to access healthcare services, ${ }^{64}$ some examples are service chains formation, institutional cooperation and coordination. Technological feasibility refers to a careful examination and a thorough assessment of the various inputs of the technologies, devices and ubiquitous environments to provide healthcare services in a nursing home setting as expected required norms. Acceptability refers to the perception, experiences and comments regarding smart nursing home from stakeholders, including the supervisory authorities and end-users (nursing home residents and caregivers).

\section{METHOD}

Based on the research questions and objectives, this scoping review comprises two phases: phase 1 provides an overview of the existing definitions, concepts and criteria of a smart nursing home through an extended search on stakeholders' websites and electronic databases; and phase 2 examines what and how technologies and medical services have been or will be implemented in a nursing home setting, and accepted by nursing home residents and caregivers. This will be done through scoping and synthesising of qualitative data from sources available on the websites of nursing homes, government agencies and the literature.

\section{Search strategy}

An extended search will be conducted using three popular search engines: 'Google', 'Yahoo' and 'Baidu (Chinese engine)' to retrieve information from websites and link the documents for definitions, concepts and criteria of a smart nursing home by any stakeholders. We will start with broad keywords such as 'nursing home', 'retirement home' and 'Yang Lao Yuan' (养老院) and then apply stricter keywords such as 'smart nursing home', 'definition of smart nursing home', 'concept of smart nursing home' and 'criteria of smart nursing home' sequentially. Search languages used are English and Chinese. If we do not find the required information on the webpage, we will extend the search to related content and documents on the same website. Within these documents (eg, reports, guidelines and regulatory documents), the search will be repeated for the above search terms. If none of the document states clear criteria for a smart nursing home, the criteria will be further refined based on the definitions and concepts. The search process will be recorded. The descriptions on the definitions, concepts and criteria of smart nursing homes from eligible articles will be extracted.

In addition, a systematic database search for published articles on technological feasibility, expectations and needs in nursing homes will be conducted. The search strategy involves a comprehensive search of a wide range of relevant databases and identification of studies in which the concept of a 'smart nursing home' is an area of research with any measure of technological feasibility, integration of medical services and acceptability of smart nursing homes by stakeholders as the outcome. A search on nine bibliographic databases: PubMed, IEEE Explore, Cochrane Library, CINAHL, Scopus, Psychology and Behavioural Sciences Collection, Health Systems Evidence, Social Systems Evidence and ProQuest Dissertations \& Theses Global will be conducted. Additionally, a search will also be conducted on two Chinese academic journals and thesis databases: the China Academic Journals Full-text Database (中国知网) and the China Dissertation Database (万方数据). These databases are chosen as they would allow the location of literature on health technologies. The keywords for online search will include Medical Subject Headings (MeSH) terms and text words 
for (A) smart healthcare technologies and (B) nursing homes for elderly people. In selecting the keywords for smart healthcare, we adopted the terms from WHO's reports and smart healthcare descriptions from published literature. The following are some examples of the keywords used: smart health*(care), IoT, digital health*, remote health*(care), telemedicine, eHealth, mobile health*(care), mHealth (including telemedicine), point-of-care, wireless sensor network, AI and ubiquitous healthcare (u-healthcare). To perform the search in the two Chinese online databases, the following keywords will be used: 'Zhi Hui Yang Lao Yuan' (智慧养老院), 'Zhi Neng Yang Lao Yuan' (智能养老院) and 'Yi Liao Kang Yang' (医疗康养). These are the common terms used in public documents issued by the Chinese government. The concept of elderly institutional healthcare and to that within the Chinese cultures refers to senior/elderly care home, elder day-care centre, senior centre, longterm care institution for elderly people, continuing care community and hospice care centre. ${ }^{65}$ The common term for this concept is 'nursing home', and it is translated as 'Yang Lao Yuan' (养老院) in Chinese. Additionally, the terms that are commonly used in other nations will be considered to be adopted in the search such as veteran nursing home (USA), old-age home (India), retirement homes (Italy) and Australian residential aged care homes (Australia). This search process will be guided by the 12-step strategy published by Kable $e t a l^{66}$ to help readers understand the process used in the search strategy. ${ }^{66}$

A detailed record of the search is documented as follows: date of search, search engine, keywords and keywords combination, number of retrieved publications and the final number of eligible studies. Online supplemental file 1 provides an example of the search strategy on PubMed.

\section{Selection of sources of evidence}

A comprehensive screening will be conducted by a reviewer (YZ) and all eligible articles will be imported to Endnotes X9 library created for this review. Duplicate articles will be removed from the library. Following this process, the Endnote library will be shared with a second reviewer (FZR or SSG) for study selection. Documents in Chinese language will be reviewed by another reviewer (BHC). Eligible criteria will be applied to both the abstracts and full texts, whichever is sufficient. Any disagreements between the two reviewers will be addressed by consulting a third reviewer. Authors may be contacted for further data and inquiry, if necessary. Figure 2 presents a flow diagram showing the articles included and excluded at each step of the selection process.

\section{Eligibility criteria}

Eligible articles that will be included in this scoping review include published quantitative, qualitative and mixed-methods articles, postgraduate theses and conference abstracts based on the Population, Intervention, Comparison, and Outcome (PICO) criteria in table 1. All sources will be included during the conceptualisation of the definition of a smart nursing home. When assessing technology feasibility, medical integration and acceptability of the technology, information from the literature gathered from the nursing home settings, nursing home residents and caregivers will be included. They must be using any type of health IT that is considered 'smart'. The literature is limited to texts written either in English and Chinese; articles involving elderly people aged 60 years old or above as residents of nursing homes, with or without some medical needs (cognitive or physical conditions) but are not terminally ill or bed-bound, and those that describe any conceptual models, frameworks and architectures of smart healthcare and those conducted in nursing home settings. The publication year is limited to those published between 1999 and 2020 as the label 'smart dust' to wireless sensor networks was first given in $1999{ }^{67}$ As one of the most important elements in the IoT paradigm, the wireless sensor networks have enabled a smart environment for healthcare services. ${ }^{68}$ In the same period, the IoT was first introduced by Ashton $\mathrm{KJRj}^{69}$ in 1999. Articles that do not examine the feasibility of smart health technology application, integration of medical services and acceptability of smart nursing home or in a non-senior care setting, for example, home-based care and hospitalisation, will be excluded.

\section{Data extraction}

YZ will extract information on the definitions, concepts and criteria of smart nursing homes from both internet sources and literature independently. Then another reviewer (BHC or SSG) will check a random of $20 \%-30 \%$ of the selected internet sources and articles. A standardised form will be created and pilot tested based on the ten articles using Excel and its usefulness will be improved accordingly. Box 1 shows the data that will be retrieved.

\section{Data analysis}

The framework method ${ }^{70}$ will be used to summarise and reduce texture data by applying codes to categorise them according to thematic analysis using combined inductive and deductive approaches. Data including internet sources will be categorised based on the type of stakeholders or organisations and the country's name. We will explore the definitions, concepts and criteria of a smart nursing home stratified by the stakeholders including care institutions, supervisory authorities, healthcare providers and the elderly people with their family members. Articles will be categorised according to the author, year of publication, study population, research site, description of definition or concept of a smart nursing home, technologies, user experience and acceptability. Moreover, recommendations for the future development of the technologies will be extracted to examine their feasibility.

On the basis of the review objectives and outcomes, data will be extracted. Respective technologies, integrated medical services and acceptability of a smart 


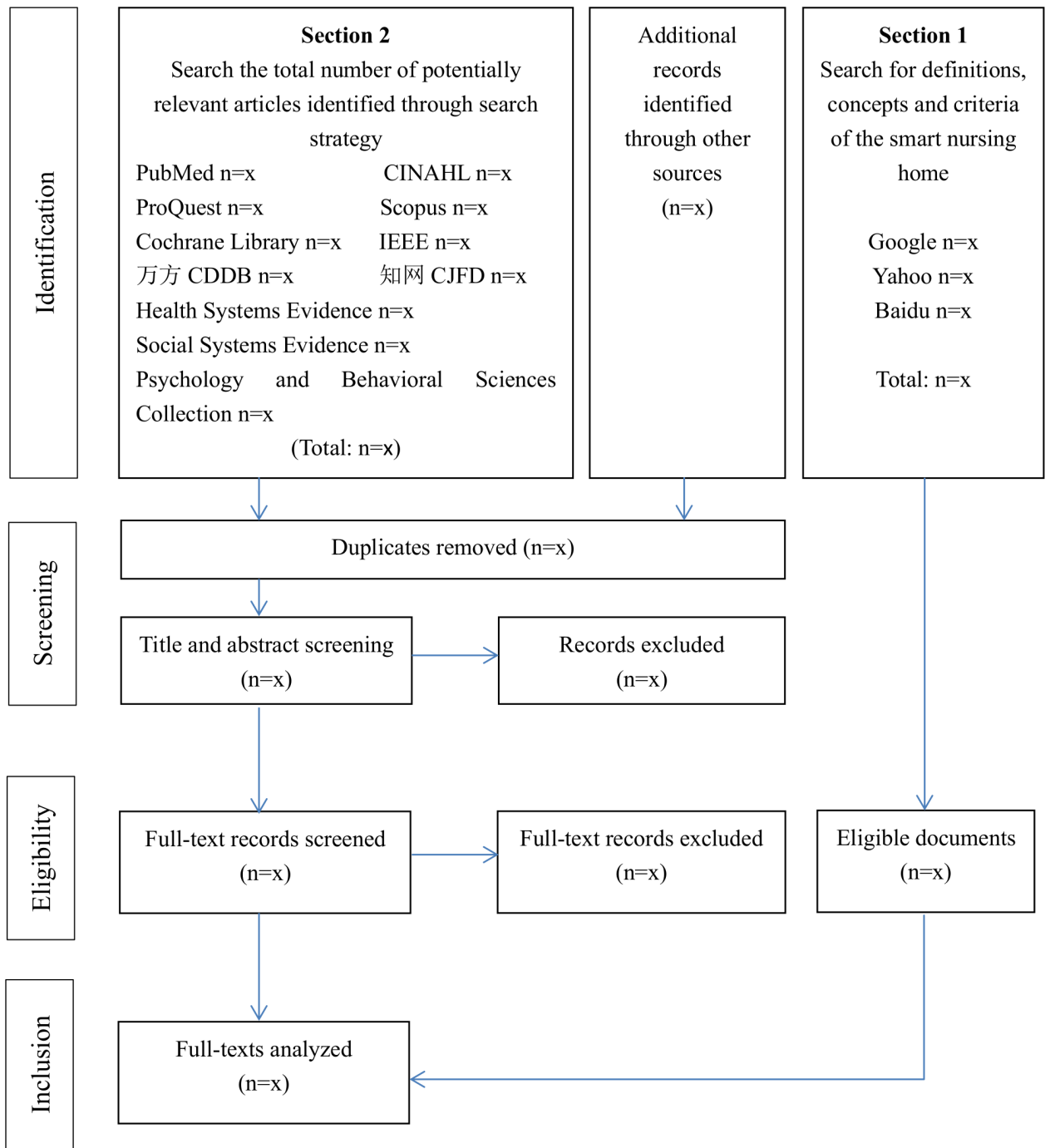

Figure 2 Scoping review flow diagram. CDDB, China Dissertation Database; CJFD, China Academic Journals Full-text Database.

\begin{tabular}{|c|c|c|}
\hline Terms & Inclusion & Exclusion \\
\hline$P$ & $\begin{array}{l}\text { 1. Nursing home residents (assessment of technology feasibility and } \\
\text { medical integration) } \\
\text { 2. Aged } 60 \text { years old or above } \\
\text { 3. With or without medical demands } \\
\text { 4. Not bed-bound }\end{array}$ & \\
\hline I & $\begin{array}{l}\text { Application of smart healthcare/respective technologies in the nursing } \\
\text { home setting }\end{array}$ & $\begin{array}{l}\text { Other technologies are irrelevant to healthcare } \\
\text { or integration of medical services, for example, } \\
\text { environmental control circuits for lighting, } \\
\text { temperature, transportation and entertainment. }\end{array}$ \\
\hline C & Not applicable & \\
\hline $\mathrm{O}$ & $\begin{array}{l}\text { 1. The conceptual models, framewors and architecture of techniques } \\
\text { of smart healthcare. } \\
\text { 2. Challenges and recommendations to implement technologies and } \\
\text { applications that facilitate medical services and nursing care. } \\
\text { 3. Perception and acceptability of smart nursing homes by the } \\
\text { elderly people, family members and other stakeholders. }\end{array}$ & \\
\hline
\end{tabular}




\section{Box 1 Items in the data extraction form}

a. Author and date of publication.

b. Type of stakeholder (individual, company/organisation or government owned).

c. Type of study (study design).

d. Country.

e. Study population (age, gender and ethnicity) and size.

f. Health conditions of the elderly people.

g. Description of definition, concept and criteria of smart nursing home.

h. Feasible technologies, function of technologies and direct user.

i. Integration of medical services.

j. Stakeholders' acceptability.

k. Notes (to capture unexpected but relevant findings).

nursing home into themes and sub-themes, will be categorised and summarised and the results will be reported qualitatively. ${ }^{71}$ Any other emerging themes will also be reported. We will apply codes to excerpts of raw data and add or modify them as new responses emerged. Codes are then grouped into clusters based on similar and interrelated ideas to identify the common and stakeholderspecific 'smart' themes in an iterative process until a consensus is reached among at least three of the four investigators (YZ, FZR, SSG and BHC). Themes will be named after the most frequently recurring terms within the same clusters; for example, generalisability, relevancy, high-quality data that are not created or imposed by the investigators. The information and data will be presented in a designed form as shown in online supplemental file 2. Online supplemental file 3 outlines the analysis plan following the framework method in six steps. Since this is a scoping review, a meta-analysis of the included studies is not applicable.

\section{CONCLUSION AND DISCUSSION}

To the best of our knowledge, this is the first scoping review to identify the scope of evidence and gaps in the literature with regard to smart nursing homes. Through the comprehensive search involving many databases and government documents, we will explore the extent, range and natural characteristics of the evidence on technological feasibility, integration of medical services and acceptability of the smart nursing home concepts and models by stakeholders, including the acceptance by the elderly people. We will define smart nursing home, categorise and analyse the technologies into the concept and application. ${ }^{72}$

We believe that the proposed scoping review will be robust enough to answer the review questions and achieve our intention to advance an evidence-based approach for a smart nursing home that is technological feasible, medical services integrated and is considered acceptable by all stakeholders. The results may be relevant to a broad base of readers interested in this research and to most industrialised countries that have nursing homes, as well as the elderly people living in them.
Although this scoping review focuses on nursing home settings, the technologies identified in smart nursing homes have potential usage in homes where ageing in place is being desirable. This scoping review may be used to compare the expressed ideas of the elderly people who have chosen a smart nursing home and those who have chosen a nursing home that does not embrace smart technologies. Additionally, the roles and influence of family members of the elderly people when making a decision in the adoption of a smart nursing home may also be identified. If resources permit, we may attempt to link quality indicators to the level of nursing home 'smartness', which would be of interest across many disciplines including health sciences, computer science, engineering and business. Future research should consider the results of this scoping review, the local cultures and government policy in order to contribute to a better smart nursing home business model, evaluation and monitoring systems that are acceptable to most stakeholders including the elderly people and their family members.

\section{Patient and public involvement}

No patients are involved in this study.

\section{Ethics and dissemination}

This protocol is registered on osf.io (URL: https:/ /osf.io/ qtwz2/). Ethical approval is not required as no primary data will be collected, and the information collected is from selected articles that are publicly available sources. The findings of the scoping review will be disseminated at conferences and published in peer-reviewed journals.

\section{Author affiliations}

${ }^{1}$ Department of Family Medicine, Faculty of Medicine and Health Sciences, Universiti Putra Malaysia, Serdang, Malaysia

${ }^{2}$ Global Century Science Group, Beijing, China

${ }^{3}$ Department of Computer and Communication Systems Engineering, Faculty of Engineering, Universiti Putra Malaysia, Seri Kembangan, Malaysia

${ }^{4}$ Malaysian Research Institute on Ageing, Universiti Putra Malaysia, Serdang, Malaysia

${ }^{5}$ Clinical Research Unit, Hospital Pengajar Universiti Putra Malaysia (HPUPM Teaching Hospital), Serdang, Malaysia

\section{Twitter Boon How Chew @chewboonhow}

Contributors YZ proposed the task and drafted the initial review protocol. All authors were involved in the design of the review. YZ will conduct the qualitative scoping review. As an expert in gerontechnology, FZR will act as the second reviewer, contributing to study selection. SSG and BHC will verify the retrieved textual data. All authors will be involved in the thematic analysis. All authors have made substantive intellectual contributions to the development of this protocol and approved the final manuscript for submission to the journal.

Funding The authors have not declared a specific grant for this research from any funding agency in the public, commercial or not-for-profit sectors.

Competing interests None declared.

\section{Patient consent for publication Not required.}

Provenance and peer review Not commissioned; externally peer reviewed.

Supplemental material This content has been supplied by the author(s). It has not been vetted by BMJ Publishing Group Limited (BMJ) and may not have been peer-reviewed. Any opinions or recommendations discussed are solely those of the author(s) and are not endorsed by BMJ. BMJ disclaims all liability and responsibility arising from any reliance placed on the content. Where the content 
includes any translated material, BMJ does not warrant the accuracy and reliability of the translations (including but not limited to local regulations, clinical guidelines, terminology, drug names and drug dosages), and is not responsible for any error and/or omissions arising from translation and adaptation or otherwise.

Open access This is an open access article distributed in accordance with the Creative Commons Attribution Non Commercial (CC BY-NC 4.0) license, which permits others to distribute, remix, adapt, build upon this work non-commercially, and license their derivative works on different terms, provided the original work is properly cited, appropriate credit is given, any changes made indicated, and the use is non-commercial. See: http://creativecommons.org/licenses/by-nc/4.0/.

\section{ORCID iDs}

Yuanyuan Zhao http://orcid.org/0000-0002-5878-5838

Fakhrul Zaman Rokhani http://orcid.org/0000-0001-6449-8184

Sazlina Shariff Ghazali http://orcid.org/0000-0002-5737-7226

Boon How Chew http://orcid.org/0000-0002-8627-6248

\section{REFERENCES}

1 IBM. Smarter planet IBM website, 2008. Available: https://www.ibm. com/ibm/history/ibm100/us/en/icons/smarterplanet/

2 WHO. Who guideline: recommendations on digital interventions for health system strengthening. Geneva: World Health Organization, 2019: P2.

3 Brown I, AAJIRoIE A. The ethical challenges of ubiquitous healthcare 2007;8:53-60.

4 Roy N, Pallapa G, Das SK. An ontology-driven ambiguous contexts mediation framework for smart healthcare applications. Proceedings of the 1st International Conference on pervasive technologies related to assistive environments. Athens, Greece: Association for Computing Machinery, 2008: Article 2.

5 Rahmani AM, Thanigaivelan NK, Gia TN. Smart e-health gateway: bringing intelligence to internet-of-things based ubiquitous healthcare systems. 2015 12th annual IEEE consumer communications and networking conference (CCNC). IEEE 2015.

6 Tian S, Yang W, Grange JML, et al. Smart healthcare: making medical care more intelligent. Glob Health J 2019;3:62-5.

7 Song I-Y, Song M, Timakum T, et al. The landscape of smart aging: topics, applications, and agenda. Data Knowl Eng 2018;115:68-79.

8 The United Nations. World population ageing 2019 United Nations; 2019. https://www.un.org/en/development/desa/population/ publications/pdf/ageing/WorldPopulationAgeing2019-Report.pdf [Accessed 04 Feb 2020].

9 WHO. Ageing and health, 2018. Available: https://www.who.int/ news-room/fact-sheets/detail/ageing-and-health

10 Bzura C, Im H, Malehorn $\mathrm{K}$. The emerging role of robotics in personal health care: bringing smart health care home [Doctoral dissertation], 2012.

11 Lv Z, et al, Xia F, Wu G, Yao L, Chen Z. iCare: a mobile health monitoring system for the elderly. 2010 IEEE/ACM Int'/ Conference on Green Computing and Communications \& Int'l Conference on Cyber, Physical and Social Computing 2010:699-705.

12 Klimova B. Elderly people and their use of smart technologies: benefits and limitations. smart education and e-learning. Berlin: Springer, 2016: 405-12.

13 Sundaravadivel P, Kougianos E, Mohanty SP. Everything you wanted to know about smart health care: evaluating the different technologies and components of the Internet of things for better health 2017;7:18-28.

14 Baker SB, Xiang W, Atkinson I. Internet of things for smart healthcare: technologies, challenges, and opportunities. IEEE Access 2017:5:26521-44.

15 WhiteHouse. Emerging technologies to support an aging population. USA: Committee on Technology of the National Science \& Technology Council, 2019

16 Marikyan D, Papagiannidis S, Alamanos E. A systematic review of the smart home literature: a user perspective. Technol Forecast Soc Change 2019;138:139-54.

17 Rantz M, Phillips LJ, Galambos C, et al. Randomized trial of intelligent sensor system for early illness alerts in senior housing. $J$ Am Med Dir Assoc 2017;18:860-70.

18 Bleda AL, Maestre R, Beteta MA. AmICare: ambient intelligent and assistive system for caregivers support. 2018 IEEE 16th International Conference on Embedded and Ubiquitous Computing 2018:66-73.

19 TheWorldBank. The world bank in China, 2018. Available: https:// www.worldbank.org/en/country/china/overview
20 GOV.cn. Law of the people's republic of China on protection of the rights and interests of the elderly: The State Council, The People's Republic of China, 2012. Available: http://www.gov.cn/flfg/2012-12/ 28/content 2305570.htm

21 Wang J. 每天发出 100 多亿养老金! 65岁以上人口 比 $11.9 \%$, 养老金 入市规模仅 15\% ? Beijing, China: 21 Jingji, 2019. Available: https:// m.21jingji.com/article/20190226/herald/e820c05844874c7b766fe4c3 3b071b0f.html

22 The United Nations. 2007年世界经济与社会概览，老龄化世界的发 展; 2007: 13. https://www.un.org/en/development/desa/policy/wess/ wess archive/2007wess ch.pdf

23 Wang LM, Chen ZH, Zhang M, et al. [Study of the prevalence and disease burden of chronic disease in the elderly in China]. Zhonghua Liu Xing Bing Xue Za Zhi 2019;40:277-83.

24 ZE Y. The main features of population aging and policy considerations in China [J]. Population \& Economics 2001;5:3-10.

25 Knapp KN. The RU Reinterpretation of Xiao. Early China 1995;20:195-222.

26 CJ S, Chen BJ. Ubiquitous community care using sensor network and mobile agent technology. 2010 7th international conference on ubiquitous intelligence \& computing and 7th international conference on autonomic \& trusted computing. IEEE 2010:99-104.

27 Robison J, Shugrue N, Porter M, et al. Transition from home care to nursing home: unmet needs in a home- and community-based program for older adults. J Aging Soc Policy 2012;24:251-70.

28 Zhang Y, FWJJoAS G. Who will care for the elderly in China?: A review of the problems caused by China's one-child policy and their potential solutions. Journal of Aging Studies 2006;20:151-64.

29 Sanford AM, Orrell M, Tolson D, et al. An international definition for "nursing home". J Am Med Dir Assoc 2015;16:181-4.

30 Serow WJ. Why the elderly move. cross-national comparisons. Res Aging 1987;9:582-97.

31 CHINADAILY. More elderly Chinese choose to live in nursing homes, 2015. Available: https://www.chinadaily.com.cn/china/2015-10/08/ content_22130227.htm

32 Chu L-W, Chi I, IJJotAMDA C. Nursing homes in China. J Am Med Dir Assoc 2008;9:237-43.

33 Xie W, Fan R. Towards ethically and medically sustainable care for the elderly: the case of China. HEC Forum 2020;32:1-12.

34 Yang M, Li P. 积极应对人口老龄化的税收政策 究. 中国科技论文在线 2008;5:84-6.

35 NPC. Law of the people's republic of China on protection of the rights and interests of the elderly', 2019. Available: http://www.npc. gov.cn/zgrdw/npc/xinwen/2019-01/07/content 2070262.htm

36 GOV.cn. Government report of healthy China 2030: the state Council, 2016. Available: http://www.gov.cn/zhengce/2016-10/25/content_ 5124174.htm

37 Demiris G, Hensel BK. Technologies for an aging society: a systematic review of "smart home" applications. Yearb Med Inform 2008; $17: 33-40$

38 Huang F, Chang P, Hou I-C, et al. Use of a mobile device by nursing home residents for long-term care comprehensive geriatric self-assessment: a feasibility study. Comput Inform Nurs 2015;33:28-36.

39 Matsui T, Yoshida Y, Kagawa M, et al. Development of a practicable non-contact bedside autonomic activation monitoring system using microwave radars and its clinical application in elderly people. J Clin Monit Comput 2013;27:351-6.

40 Pulli P, Hyry J, Pouke M, et al. User interaction in smart ambient environment targeted for senior citizen. Med Biol Eng Comput 2012;50:1119-26.

41 Pallikonda Rajasekaran M, Radhakrishnan S, Subbaraj P. Sensor grid applications in patient monitoring. Future Generation Computer Systems 2010;26:569-75

42 Mieronkoski R, Azimi I, Rahmani AM, et al. The Internet of things for basic nursing care-a scoping review. Int J Nurs Stud 2017;69:78-90.

43 Yuehong $\mathrm{Y}$, Zeng $\mathrm{Y}$, Chen $\mathrm{X}$. The Internet of things in healthcare: an overview. Journal of Industrial Information Integration 2016;1:3-13.

44 Mohammed J, Lung $\mathrm{CH}$, Ocneanu A. Internet of things: remote patient monitoring using web services and cloud computing. 2014 IEEE international conference on internet of things (IThings), and IEEE green computing and communications (GreenCom) and IEEE cyber, physical and social computing 2014:256-63.

45 Raja T. Internet of things: benefits and risk of smart healthcare application. Innovation 2016;10:37-42.

$46 \mathrm{Zhu} \mathrm{H}$, Wu CK, Koo CH, et al. Smart healthcare in the era of Internetof-Things. IEEE Consumer Electron Mag 2019;8:26-30.

47 Golant SM, SMJJoAS G. A theoretical model to explain the smart technology adoption behaviors of elder consumers (Elderadopt). $J$ Aging Stud 2017;42:56-73. 
48 Dong B, Wu S. Nursing homes in China: now and the future, 2010. Available: https://docplayer.net/16943513-Nursing-homes-in-chinanow-and-the-future.html

49 Tricco AC, Lillie E, Zarin W, et al. PRISMA extension for scoping reviews (PRISMA-ScR): checklist and explanation. Ann Intern Med 2018;169:467-73.

50 Kang B, Scales K, McConnell ES, et al. Nursing home residents' perspectives on their social relationships. J Clin Nurs 2020;29:1162-74.

51 McDerby N, Bail K, Kosari S, et al. Canaries in the coalmine: Stakeholder perspectives of medication management reviews for residents with dementia. Res Social Adm Pharm 2020;16:1220-7.

52 Shune SE, Linville D, DJljons L. Understanding the dining experience of individuals with dysphagia living in care facilities: a grounded theory analysis. Int J Nurs Stud 2019;92:144-53.

53 Jain B, Willoughby M, Winbolt M, et al. Stakeholder perceptions on resident-to-resident aggression: implications for prevention. Aust Health Review 2018;42:680-8.

54 Stocker R, Bamford C, Brittain K, et al. Care home services at the vanguard: a qualitative study exploring stakeholder views on the development and evaluation of novel, integrated approaches to enhancing healthcare in care homes. BMJ Open 2018;8:e017419.

55 Rhynas SJ, Garrido AG, Burton JK, et al. New care home admission following hospitalisation: how do older people, families and professionals make decisions about discharge destination? A case study narrative analysis. Int J Older People Nurs 2018;13:e12192.

56 Scales K, Lepore M, Anderson RA, et al. Person-directed care planning in nursing homes: resident, family, and staff perspectives. $J$ Appl Gerontol 2019;38:183-206.

57 Millar AN, Daffu-O'Reilly A, Hughes CM, et al. Development of a core outcome set for effectiveness trials aimed at optimising prescribing in older adults in care homes. Trials 2017;18:175.

58 Cooper E, Spilsbury K, McCaughan D, et al. Priorities for the professional development of registered nurses in nursing homes: a Delphi study. Age Ageing 2017;46:39-45

59 Goodman C, Davies SL, Gordon AL, et al. Relationships, expertise, incentives, and governance: supporting care home residents' access to health care. An interview study from England. J Am Med Dir Assoc 2015;16:427-32.
60 Schneider J, Dening T. Effective health care for older people resident in care homes: the optimal study protocol for realist review. Syst Rev 2014;3:1-7.

61 Miller EA, Rudder C. Engaging consumers in Medicaid nursing home reimbursement policy: lessons from New York and Minnesota. Gerontologist 2013;53:627-40.

62 Iris M, DeBacker NA, Benner R, et al. Creating a quality of life assessment measure for residents in long term care. J Am Med Dir Assoc 2012;13:438-47.

63 Mei YY, Marquard J, Jacelon C, et al. Designing and evaluating an electronic patient falls reporting system: perspectives for the implementation of health information technology in long-term residential care facilities. Int J Med Inform 2013;82:e294-306.

64 Botezat D, Oprea L, Iliescu R. Integrating medical services: a solution for an efficient and equitable health system. Revista Romana de Bioetica 2013;11:93-107.

65 Zhan HJ, Luo B, Chen Z. Institutional elder care in China. Aging in China. Boston, MA: Springer, 2012: 221-35.

66 Kable AK, Pich J, Maslin-Prothero SE. A structured approach to documenting a search strategy for publication: a 12 step guideline for authors. Nurse Educ Today 2012;32:878-86.

67 Kahn JM, Katz RH, Pister KS. Next century challenges: mobile networking for "Smart Dust". Proceedings of the 5th annual ACM/ IEEE international conference on Mobile computing and networking 1999:271-8.

68 Piyare R, Lee SR, SRJapa L. Towards Internet of things (iots): integration of wireless sensor network to cloud services for data collection and sharing. IJCNC 2013;5:59-72.

69 Ashton KJRj. That 'internet of things' thing. RFID journal 2009;22:97-114.

70 Gale NK, Heath G, Cameron E, et al. Using the framework method for the analysis of qualitative data in multi-disciplinary health research. BMC Med Res Methodol 2013;13:117.

71 Ritchie J, Lewis J, Nicholls CM. Qualitative research practice: a guide for social science students and researchers. Thousand Oaks, California: Sage, 2013.

72 Schiefer M. Smart home definition and security threats. 2015 ninth international conference on IT security incident management \& IT forensics. IEEE 2015:114-8. 